Pacific Journal of Mathematics

THE NUMBER OF AUTOMORPHISMS OF AN ATOMIC 


\title{
THE NUMBER OF AUTOMORPHISMS OF AN ATOMIC BOOLEAN ALGEBRA
}

\author{
JUDY ROITMAN
}

A method of construction via forcing is developed which allows great freedom in the interplay among the number of atoms, number of automorphisms, size of the algebra, and such objects of settheoretic interest as $c$. As by-products we have

Theorem 1. The following is consistent: there is a 0dimensional Hausdorff space with fewer than $c$ autohomeomorphisms, at least one of which moves a nonisolated point.

Theorem 2. The following is consistent: there is an infinite Boolean algebra with more automorphisms than elements, the number of whose automorphisms is not a power of 2.

o. Introduction. This paper explores the interplay among the number of atoms, number of automorphisms, and size of an atomic Boolean algebra, and the relation of these cardinals to such set theoretic objects as $c$. As by-products we have

THEOREM 1. The following is consistent: there is a 0-dimensional Hausdorff space with fewer than c automorphisms, at least one of which moves a nonisolated point.

THEOREM 2. The following is consistent: there is an infinite Boolean algebra with more automorphisms than elements, the number of whose automorphisms is not a power of 2 .

Background. De Groot and McDowell had shown that a regular Hausdorff space has uncountably many automorphisms if at least one nonisolated point is moved by some automorphism. Their proof suggested that "uncountably many" might be pushed to "at least $c$ many," but Theorem 1 refutes this.

Theorem 2 is a partial answer to question 4 of the triple survey paper [2]. We'll say that a Boolean algebra is rich if the number of automorphisms is greater than its size. If $\lambda \leqq \kappa<2^{\lambda}$ it's easy to find rich algebras of size $\kappa$ with $2^{\lambda}$ automorphisms. Must all rich algebras be of this type? Theorem 2 says no. What cardinals are possible for the number of automorphisms of a rich algebra? Theorem 3(c), below, gives a wide range of possibilities.

The main theorem of this paper is the result of an investigation of how a construction of van Douwen could be generalized, altered, 
and eventually mauled in order to construct, in certain models of set theory, atomic Boolean algebras with various properties which demonstrate that certain cardinal invariants have few restraints. We need some terminology to state these results.

An almost rigid algebra is one in which no automorphism moves infinitely many atoms. Define $\boldsymbol{P}_{\eta}(\rho)$ as the set of functions from $\rho$ into 2 of size $<\eta$. Recall that if $M$ is a model of set theory and $\eta^{<\eta}=\eta$ in $M$ then forcing with $P_{\eta}(\rho)$ over $M$ preserves cardinals. As ad hoc terminology, say that a triple $(\lambda, \gamma, \kappa)$ is realized if there is a Boolean algebra with a dense set of atoms for which $\lambda=$ number of atoms, $\gamma=$ number of automorphisms, and $\kappa=$ cardinality.

THEOREM 3. Let $M$ be a model of set theory in which $\eta^{<\eta}=\eta$ and let $N=M^{P_{\eta}(\rho)}$. The foffwing hold in $N$ :

(a) if $\omega \leqq \eta \leqq \lambda<\rho$ then $(\lambda, \lambda, \kappa)$ is realized in an almost rigid algebra for any $\kappa \in\left[\lambda^{+}, 2^{\lambda}\right]$.

(b) if $\eta \leqq \lambda<\rho, \lambda \leqq \gamma \leqq \kappa \leqq 2^{\lambda}$, and $(\lambda \neq \kappa)$, then $(\lambda, \gamma, \kappa)$ is realized a not almost rigid algebra.

(c) if $\eta \leqq \lambda<\rho$ and, for some $\sigma, \lambda \leqq \kappa<\gamma=\lambda^{\sigma}$, then $(\lambda, \gamma, \kappa)$ is realized.

Part (c) generalizes further, but to state it now would just have made it even less accesible to the reader. It is stated in full generality in $\S 5$.

We flesh out Theorem 3 by an example which gives Theorem 2 as an immediate corollary. Here's a sample of what happens when you add $\aleph_{\omega_{1}+1}$ subsets of $\omega_{2}$ via $\omega_{2}$-closed conditions to a model of GCH (here $\left.\eta=\omega_{2}, \rho=\aleph_{\omega_{1}+1}\right)$ :

From 1(a) an almost rigid atomic Boolean algebra with $\boldsymbol{\aleph}_{2}$ atoms may have size $\boldsymbol{\aleph}_{\alpha}$ for any $\alpha \in\left[3, \omega_{1}+1\right]$.

From $1(b)$ there is a plethora of realized triples. For example, there is an atomic Boolean algebra of size $\boldsymbol{\aleph}_{\omega_{1}}$ with $\boldsymbol{\aleph}_{17}$ atoms and $\aleph_{\omega}$ automorphisms.

From 1(c) there is an atomic Boolean algebra of size $\aleph_{\omega}$ with $\boldsymbol{\aleph}_{\omega}$ atoms and $\boldsymbol{\aleph}_{\omega+1}$ automorphisms. This proves Theorem 2 since, in $N, 2^{\omega_{1}}<\left(\boldsymbol{\aleph}_{\omega}\right)^{\omega}=\boldsymbol{\aleph}_{\omega+1}<2^{\omega_{2}}$.

Here's how to prove Theorem 1 from the constructions of Theorem 3. Add at least $\omega_{2}$ Cohen reals to any model of set theory so $\mathrm{CH}$ doesn't hold. By Theorem $1(\mathrm{~b})$ there is an atomic Boolean algebra with countably many atoms and exactly $\omega_{1}$ automorphisms. Inspecting the construction shows that some automorphism moves some nonatomic element to an incompatible element. By Stone duality this means the Stone space has exactly $\omega_{1}$ automorphisms, at least one of which moves a nonisolated point. 
The paper is organized as follows: $\S 1$ gives preliminaries, mostly set-theoretic. $\S 2$ constructs almost rigid algebras to prove Theorem 3(a). $\$ 3$ gives canonical forms for Boolean algebras used in $\S 4$, which gives the simplest nonalmost rigid construction. $\S 5$ uses a direct limit of previous constructions to complete the proofs of $3(b)$ and $(c)$.

I thank Jim Loats for introducing me to these questions and stimulating my interest in them.

1. Preliminaries. All algebras in this paper are Boolean algebras with an infinite dense set of atoms. Thus without loss of generality an algebra $B$ is a subalgebra of some $\mathscr{P}(A)$, where $A$ is an infinite set of ordinals, and contains all finite and cofinite subsets of $A$. We say $B$ is an algebra on $A$ and occasionally identify the atom $\{\alpha\}$ with the element $\alpha$-this will be clear from the context.

$A$ ! is the set of all permutations of $A$. For $f \in A !, x \subset A$, we write $f(x)=\{f(\alpha): \alpha \in x\}$. Thus $A$ ! is identified with the full set of automorphisms of the algebra $\mathscr{P}(A)$. If $B$ is an algebra on $A, \Psi$ an automorphism of $B$, and $f \in A$ ! is defined by $f(\alpha)=\Psi(\{\alpha\})$, then $f(x)=\Psi(x)$ for all $x \in B$. Thus the automorphism group of an algebra is identified with a subgroup of $A$ !

Let $\eta$ be an infinite cardinal. An ordinal $\alpha$ is $\eta$-finite iff $\alpha<\eta$; otherwise it is $\eta$-infinite. A set $A$ is $\eta$-finite iff $|A|<\eta$, where $|A|=$ cardinality of $A$. If $A$ is not $\eta$-finite it is $\eta$-infinite.

$[A]^{<\eta}$ is the collection of all $\eta$-finite subsets of $A$.

${ }^{4} 2$ is the set of functions from $A$ into $2 ; 2^{A}=\left|{ }^{A} 2\right|$.

Ordinals are denoted by lower-case Greek letters. The letters $\lambda, \gamma, \kappa, \rho, \eta, \zeta, \tau$ are reserved for cardinals.

All models in this paper are countable transitive models of ZFC. We say the model $N$ is generated by an incresing sequence of models $\left\{M_{\alpha}: \alpha<\rho\right\}$ iff for some $\left\{y_{\alpha}: \alpha<\rho\right\} \subset V$, each $M_{\alpha}=M_{0}\left[\left\{y_{\beta}: \beta<\alpha\right\}\right]$ and $N=M_{0}\left[\left\{y_{\alpha}: \alpha<\rho\right\}\right]$. If $M$ is a model and $\boldsymbol{P}$ a partial order, $M^{P}$ is the Boolean valued model gotten by forcing with $\boldsymbol{P}$ over $M$.

Let $A$ be $\eta$-infinite. The $\eta$-closed partial order on $A, \boldsymbol{P}_{\eta}(A)$, is the set of $\eta$-finite functions from $A$ into 2 under the order of reverse inclusion. Note that $\boldsymbol{P}_{\omega}(A)$ is just the usual Cohen partial order on $A$. A set $x \subset A$ is an $\eta$-Cohen subset of $A$ over a model $M$ iff its characteristic function is the union of an $M$-generic filter on $\boldsymbol{P}_{\eta}(A) \cap M$.

Fact 1.1 (preservation of $\eta$-Cohen objects). Let $M$ be a model in which $A^{\prime}$ is $\eta$-infinite and suppose $x$ is an $\eta$-Cohen subset of $A^{\prime}$ over $M$.

(1) $A^{\prime}-x$ is an $\eta$-Cohen subset of $A^{\prime}$ over $M$. 
(2) if $A \in M$ is an $\eta$-infinite subset of $A^{\prime}$, then $x \cap A$ is an $\eta$-Cohen subset of $A$ over $M$.

(3) if $f: A^{\prime} \rightarrow A$ is in $M, f$ is onto, each $f^{-1}(\alpha)$ is $\eta$-finite, and $A$ is $\eta$-infinite, then $f(x)$ is an $\eta$-Cohen subset of $A$ over $M$.

We remind the reader of some elementary facts about forcing with $\boldsymbol{P}_{\eta}(\rho)$ over a model $M$ (we understand this to mean forcing with $\left.\boldsymbol{P}_{r}(\rho) \cap M\right)$ :

Fact 1.2 (model structure). Let $N=M^{P_{\eta}(\rho)}$.

(i) $N$ adds no new $\eta$-finite sets of ordinals to those in $M$.

(ii) if $\eta^{<\eta}=\eta$ in $M$, cardinals are not collapsed.

(iii) if $A A \in M$ and $|A|=\rho$ in $M$ then $\boldsymbol{P}_{\eta}(\rho)$ is isomorphic to $\boldsymbol{P}_{\eta}(A)$.

(iv) homogeneity: let $x \in N$ be an $\eta$-Cohen subset of $A \subset \rho$ over $M$. Then is $\eta$-Cohen over $M^{\boldsymbol{P}_{\eta}(\rho-A)}$.

(v) flexible iteration: let $|\lambda \cdot \zeta| \leqq \rho$ and $\eta \leqq \lambda$. Then forcing with $\boldsymbol{P}_{\eta}(\rho)$ gives a model generated by an increasing sequence of models $\left\{M_{\alpha}: \alpha<\zeta\right\}$ where $M=M_{0}$ and each $M_{\alpha+1}$ has an element which is an $\eta$-Cohen subset of $\lambda$ over $M_{\alpha}$. Furthermore, if $\eta \cdot \tau<\zeta$ then any subset of $\tau$ in $N$ is in some $M_{\alpha}$. (Note that $N$ is generated by many different sequences.)

An immediate application of 1.2 (iv) is that forcing with $\boldsymbol{P}_{\eta}(\rho)$ once is the same as forcing with it twice, that is, if $\boldsymbol{P}=\boldsymbol{P}_{\eta}(\rho)$ then $M^{P}$ is isomorphic to $\left(M^{P}\right)^{P}$. Thus we may assume $M=\left(M^{*}\right)^{P}$ for some $M^{*}, N=M^{P}$, and hence for all $\tau,\left(2^{\tau}\right)^{M}=\left(2^{\tau}\right)^{N}$. In particular, in the hypothesis of Theorem 3 we may assume that the cardinalities of power sets are preserved: for all $\tau,\left(2^{\tau}\right)^{M}=\left(2^{\tau}\right)^{N}$. This gives rise to the following convenient assumption, which will be used in most of the later propositions:

$C A: \quad \boldsymbol{P}=\boldsymbol{P}_{\eta}(\rho)$ for some $\eta$ and $\rho ; M$ is a model in which $\eta^{<\eta}=\eta$; $N=M^{P}$; and for all $\tau,\left(2^{\tau}\right)^{M}=\left(2^{\tau}\right)^{N}$.

Our proofs will be given in the framework of $C A$, although other iterated models with enough $\eta$-Cohen objects would do for most of our constructions.

There is a useful combinatorial definition of $\eta$-Cohen sets, whose invocation in this paper will be called the usual Cohen argument (TUCA): $x$ is an $\eta$-Cohen subset of $A$ over $M$ iff its characteristic function meets every $M$-coded dense open subset of the space $\left.\square<{ }_{<r}{ }^{A} 2\right)$, which is the space whose underlying set is ${ }^{A} 2$ and whose basis is all $U_{g}=\{f: f \supset g\}$ where $g \in \boldsymbol{P}_{\eta}(A)$. We will use TUCA mostly in the following two forms:

Assume $M$ is a model, $A$ is $\eta$-infinite in $M, x$ is an $\eta$-Cohen subset of $A$ over $M$.

I (finite form). If $S \in M$ is an $\eta$-infinite collection of finite 1-1 
functions from some $n<\omega$ into $A$ and $a \subset n$, then $S$ has a subset $S^{\prime}$ of the same cardinality as $S, S^{\prime} \in M[x]$, where for all $i<n$ and all $s \in S^{\prime}, i \in a$ iff $s(i) \in x$.

II (infinite form). If $S \in M$ is an $\eta$-infinite collection of $\eta$-finite functions from $A$ into 2 with disjoint domains, then $S$ has a subset $S^{\prime}$ of the same cardinality as $S, S^{\prime} \in M[x]$, so that if $f \in S^{\prime}$ and $\alpha \in$ $\operatorname{dom} f$ then $\alpha \in x$ iff $f(\alpha)=0$.

We will also use the following density argument: Assume CA, where $N$ is generated by $\left\{M_{\alpha}: \alpha<\zeta\right\}$ as in (v) and each $M_{\alpha+1}$ adds an $\eta$-Cohen subset of $\tau, x_{\alpha}$, to $M_{\alpha}$. Suppose $\eta<c f(\zeta)$. Then if $f$ is an $\eta$-finite function from $\tau$ into $2,\{\alpha<\zeta$ : for all $\beta \in \operatorname{dom} f(f(\beta)=$ 0 iff $\left.\left.\beta \in x_{\alpha}\right)\right\}$ is cofinal in $\zeta$.

2. Almost rigid algebras. The task here is to construct an algebra $B$ on $\lambda$ so that if $g \in \lambda$ ! moves infinitely many atoms then there is a generic $x \in B$ so $g(x) \notin B$. In order to manipulate the elements of our algebra we need the following

Canonical form. Let $B$ be an algebra generated by a family of sets $\mathscr{S}$ and for $x \in \mathscr{S}$ let $B_{x}$ be the subalgebra of $B$ generated by $\mathscr{S}-\{x\}$. Then every element of $B$ has the form $(b \cap x) \cup(c \sim x)$ for some $b, c \in B_{x}$.

Proposition 2.1. Assume CA. Let $\eta \leqq \lambda<\rho$ and suppose $\kappa \epsilon$ $\left[\lambda^{+}, 2^{\lambda}\right]$. Then there is an almost rigid algebra of size $\kappa$ with $\lambda$ atoms.

Proof. By $1.2(\mathrm{v}), N$ is generated by an increasing sequence of models $\left\{M_{\alpha}: \alpha<\lambda^{+}\right\}$where each $M_{\alpha+1}$ has in it an $\eta$-Cohen subset $x_{\alpha}$ of $\lambda$ over $M_{\alpha}$, and each subset of $\lambda$ in $N$ is in some $M_{\alpha}$. Since $\left(2^{\lambda}\right)^{M}=\left(2^{\lambda}\right)^{N}$ we let $B_{0} \in M_{0}$ be an algebra on $\lambda$ of size $\kappa$ so that every infinite $b \in B_{0}$ has size $\lambda$. For $a<\lambda^{+}$let $B_{\alpha}$ be the algebra generated by $\left\{x_{\beta}: b<\alpha\right\}$. Notice that $B_{\alpha} \in M_{\alpha+1}$ and $B_{\alpha} \subset M_{\alpha}$. Let $B=\bigcup_{\alpha<\lambda^{+}} B_{\alpha}$. We show that $B$ is amost rigid.

Fix $g \in \lambda ! \cap N$ where $g$ moves infinitely many atoms. We are done if we can find a $\beta$ so $g\left(x_{\beta}\right) \notin B$. For some fixed $\alpha, g \in M_{\alpha}$. So if $\beta \geqq \alpha, g\left(x_{\beta}\right) \in M_{\beta+1}$, hence if $g\left(x_{\beta}\right) \in B$, then $g\left(x_{\beta}\right) \in B_{\beta+1}$.

Suppose $g$ moves infinitely many atoms, bnt fewer than $\eta$ of them. By induction on the $B_{\beta}$ 's and using the infinite case of TUCA, every infinite element of $B$ has size $\lambda$. So if any $g\left(x_{\beta}\right) \in B$ then $g\left(x_{\beta}\right)=x_{\beta}$.

Let $A \subset \lambda$ be infinite where $g^{-1}(A) \cap A=\varnothing$. By assumption, $A$ is $\eta$-finite. Letting $f$ be the function which is constantly 0 on $A$ and constantly 1 on $g^{-1}(A)$, by the density argument there is some $\beta \geqq \alpha$ 
so that for all $\alpha \in A, \alpha \in x_{\beta}$ iff $\alpha \notin g\left(x_{\beta}\right)$. But then $g\left(x_{\beta}\right) \neq x_{\beta}$ and we are done.

Now suppose $g$ moves an $\eta$-infinite set of atoms. Let $d \in B_{\alpha+1}$. By the canonical form

$$
d=\left(b \cap x_{\alpha}\right) \cup\left(c \sim x_{\alpha}\right)
$$

where $b, c \in B_{\alpha}$. Let $E \in M_{\alpha}$ be a set of size $\eta$ so that $g(E) \cap E=\varnothing$. There are four cases needed to show that $g\left(x_{\alpha}\right) \neq d$.

Case 1. $b \cap c \cap g(E)$ is $\eta$-infinite. Then there is an $\eta$-infinite collection in $M(\alpha)$ of pairs $\langle\delta, g(\delta)\rangle$ where $g(\delta) \in b \cap c \cap g(E)$. By the finite case of TUCA there are $\eta$ many of these pairs for which $\delta \notin x_{\alpha}$, so $b \cap c \cap g(E) \sim g\left(x_{\alpha}\right)$ is $\eta$-infinite. But $d \cap b \cap c \cap g(E)=b \cap c \cap g(E)$, so $d \sim g\left(x_{\alpha}\right)$ is $\eta$-infinite, and $g\left(x_{\alpha}\right) \neq d$.

Case 2. $(b \sim c) \cap g(E)$ is $\eta$-infinite. Then there is an $\eta$-infinite collection in $M(\alpha)$ of pairs $\langle\delta, g(\delta)\rangle$ where $g(\delta) \in(b \sim c) \cap g(E)$. By the finite case of TUCA there are $\eta$-many of these pairs for which $g(\delta) \in(b \sim c) \cap g(E)$ and $\delta \notin x_{\alpha}$. So $(b \sim c) \cap g(E) \sim g\left(x_{\alpha}\right)$ is $\eta$-infinite. But $(d \sim c) \cap g(E)=(b \sim c) \cap g(E)$, so $d \sim g\left(x_{\alpha}\right)$ is $\eta$-infinite, and $g\left(x_{\alpha}\right) \neq d$.

Case 3. $(c \sim b) \cap g(E)$ is $\eta$-infinite. Then there is an $\eta$-infinite collection in $M_{\alpha}$ of pairs $\langle\delta, g(\delta)\rangle$ where $g(\delta) \in(c \sim b) \cap g(E)$. By the finite case of TUCA there are $\eta$-many pairs $\langle\delta, g(\delta)\rangle$ where $\delta \in x_{\alpha}$ and $g(\delta) \in(c \sim b) \cap g(E)$. So $(c \sim b) \cap g(E) \cap g\left(x_{\alpha}\right)$ is infinite. But $d \cap(c \sim b)$ is contained in $\lambda \sim g\left(x_{\alpha}\right)$, so $g\left(x_{\alpha}\right) \sim d$ is infinite, and $g\left(x_{\alpha}\right) \neq d$.

Case 4. $g(E) \sim(b \cup c)$ is $\eta$-finite. By the infinite form of TUCA, if $g(E) \sim(b \cup c)$ is $\eta$-infinite, so is $g\left(x_{\alpha}\right) \sim d$.

Thus $g\left(x_{\alpha}\right) \notin B_{\alpha}$ and we have proved Proposition 2.1. By the remarks after 1.2 , this also proves Theorem $3(\mathrm{a})$.

3. More canonical forms. In trying to construct algebras which are not almost rigid we want to carefully control the set of automorphisms. This task is complicated by the fact that adding to an algebra generally adds to its automorphism group, as the following lemma implicit in [3] makes clear.

LEMMA 3.1. Let $B$ be an algebra and suppose there is an infinite $b \in B$ and an automorphism $f$ of $B$ where $f(b) \cap b=\varnothing$. If $c \in B$ is a subset of $b$, then $f_{c}$ is an automorphism of $B$, where 


$$
f_{c}(\alpha)= \begin{cases}f(\alpha) & \text { if } \quad \alpha \in c \\ f^{-1}(\alpha) & \text { if } \alpha \in f(c) \\ \alpha & \text { otherwise. }\end{cases}
$$

By 3.1 an automorphism moving infinitely many atoms usually carries along with it a whole family of automorphisms, whose members are essentially the same. In the next section we will construct algebras for which these are the only automorphisms. For ease of proof we require the original function $f$ to be especially well-behaved.

Definition 3.2. A function $f \in A$ ! is nice if it is its own inverse and is nowhere the identity. If $f \in A$ ! is nice and $b \subset A$ we define $f_{b} \in A$ ! by

$$
f_{b}(\alpha)= \begin{cases}f(\alpha) & \text { if } \alpha \in b \cup f(b) \\ \alpha & \text { otherwise }\end{cases}
$$

Then $f_{b}$ is also a self-inverse element of $A$ ! and is defined consistently with 3.1 .

For the remainder of this section fix $A$ a set of ordinals and some nice $f \in A$ ! We say an algebra $B$ on $A$ is closed under $f$ iff for each $b \in B, f_{b}$ is an automorphism of $B$.

Suppose $\mathscr{S}$ generates an algebra $C$ on $A$ and $B$ is the closure of $C$ under $f$. Echoing the canonical form of $\S 2$ we say $C_{x}$ is the algebra generated by $\mathscr{S}-\{x\}$. Abusing this notation we say $B_{x}$ is the closure of $C_{x}$ under $f$. By elementary set theory, $B=\left\{f_{c}(b)\right.$ : $b, c \in B\}$. So by the canonical form of $\S 2$ and the distributivity of $f$ over Boolean operations, each element of $B$ is the finite union of terms where each term has one of the following forms (in all forms $\left.b \in B_{x}\right)$ :

Form 1. $b$

Form 2. $b \cap x$

Form 3. $b \sim x$

Form 4. $\quad b \cap f(x)$

Form 5. $\quad b \sim f(x)$

Form 6. $b \cap x \cap f(x)$

Form 7. $b \cap x \sim f(x)$

Form 8. $b \cap(f(x) \sim x)$

Form 9. $\quad b \sim(x \cup f(x))$.

If $c$ is such a term we call $b$ the $B_{x}$-part of the term and write $b=c^{*}$.

By further elementary calculations we may also assume that each element of $B$ is some $\bigcup_{i \leqq q} b_{i}$ where 
(a) either $b_{\imath}=\varnothing$ or $b_{i}$ has form $i$.

(b) if $i \leqq 5$ and $j \geqq 6$ then $b_{i}^{*} \cap b_{j}^{*}=\varnothing$.

(c) $b_{1}$ is disjoint from all other $b_{j}^{*}$.

(d) $b_{2}^{*} \cap b_{3}^{*}=\varnothing$.

(e) $b_{4}^{*} \cap b_{5}^{*}=\varnothing$.

(f) $\bigcap_{i=6}^{9} b_{i}^{*}=\varnothing$.

This leads to the $f-x$ canonical form: if $b \in B$ then $b=\bigcup_{i \unlhd q} b_{i}$ where each nonempty $b_{i}$ has form $i$, and properties (a) through (f) hold. We write $b^{*}=\bigcup_{i \leqq 9} b_{i}^{*}$. Note that $b \subset b^{*}$.

4. Essentially one nontrivial automorphism. If $B$ is an algebra on $A$ and $f \in A$ ! in nice, we define $\mathscr{F}(f, b)=\left\{f_{b}: b \in B\right\}$. The task of this section is to construct an algebra $B$ whose automorphism class is exactly some $\mathscr{F}(f, B)$.

Proposition 4.1. Assume CA. Let $\eta \leqq \lambda<\rho$ and suppose $\gamma \in\left[\lambda^{+}, 2^{\lambda}\right]$. Let $f \in \lambda ! \cap M$ be nice. Then in $N$ there is an algebra $B$ on $\lambda$ of size $\gamma$ whose automorphism group is exactly $\mathscr{F}(f, b)$. Hence $(\lambda, \gamma, \gamma)$ is realized.

Proof. As in 2.1 we may assume $N$ is generated by $\left\{M_{\alpha}: \alpha<\lambda^{+}\right\}$ where $M=M_{0}$ and for all $\alpha<\lambda^{+}$there is $x_{\alpha} \in M_{\alpha+1}$ which is an $\eta$ Cohen subset of $\lambda$ over $M_{\alpha}$. Let $B_{0} \in M_{0}$ be an $f$-closed algebra on $\lambda$ of size $\gamma$ where each infinite $b \in B_{0}$ has size $\lambda$. For $\alpha<\lambda^{+}$let $B_{\alpha}$ be the smallest $f$-closed algebra containing $B_{0} \cup\left\{x_{\beta}: b<\alpha\right\}$ and let $B=\mathrm{U}_{\alpha<\lambda^{+}} B_{\alpha}$. Then $B$ has size $\gamma$. We show that its automorphism class is exactly $\mathscr{F}(f, B)$.

By the preservation facts of $\S 1$, the $f-x$ canonical form, TUCA, and induction on the $B_{\alpha}$ 's, each infinite element of $B$ has size $\lambda$. Recall from $\S 1$ that if $A, A^{\prime}$ are disjoint $\eta$-finite element of $\lambda$ then for cofinally many $\alpha<\lambda^{+}, A \subset x_{\alpha}$ and $A \cap x_{\alpha}=\varnothing$. So the argument in 2.1 shows that if $g \in \lambda$ ! and $g$ differs from some element of $\mathscr{F}(f, B)$ on exactly an $\eta$-finite set, then $g$ is not an automorphism of $B$.

So fix $g \in \lambda ! \sim \mathscr{F}(f, B)$ and assume $g$ differs from each element of $\mathscr{F}(f, B)$ on an $\eta$-infinite set. Then $g \in M_{\alpha}$ for some $\alpha$, and as in 2.1 if $g\left(x_{\alpha}\right) \in B$ then $g\left(x_{\alpha}\right) \in B_{\alpha+1} \sim B_{\alpha}$. So let $b \in B_{\alpha+1} \sim B_{\alpha}$ and suppose $b$ has the $f-x_{\alpha}$ canonical form $\bigcup_{i} b_{\imath}$ (where $\mathscr{S} \subset\left\{x_{\beta}: \beta \leqq \alpha\right\}$ ). We show $g\left(x_{\alpha}\right) \neq b$.

Define the function is on $\lambda$ by

$$
\dot{\psi}(\delta)=\left\{i: g(\delta) \in b_{i}^{*}\right\}
$$

and let $E=\{\delta: g(\delta) \neq f(\delta)\}, F=\{\delta: g(\delta) \neq \delta\}$. Since $f$ is nowhere the identity, $\lambda=E \cup F$. There are two cases: $E \cap F$ is $\eta$-infinite and 
$E \cap F$ is $\eta$-finite.

Case 1. $E \cap F$ is $\eta$-infinite. Note that $E \cap F \in M_{\alpha}$. If $g(E \cap F) \sim b^{*}$ is $\eta$-infinite then by TUCA $g\left(x_{\alpha}\right) \sim b$ is $\eta$-infinite and we are done. So assume $b^{*} \cap g(E \cap F)$ is $\eta$-infinite. Then there is an $\eta$-infinite $H \in M_{\alpha}$ and a finite $s \in M_{\alpha}$ so $H \subset E \cap F, g(H) \subset b^{*}$, and $\psi(g(\delta))=s$ for all $\delta \in H$.

Now the argument divides into subcases and subsubcases, all of which exploit the fact that if $\delta \in H$ then no two of $\delta, g(\delta)$ or $f g(\delta)$ are equal.

Subcase 1(a). $s \subset 6$. If $s=\{1\}$ then $g(H) \subset b_{1}$ and by TUCA both $b \sim g\left(H \cap x_{\alpha}\right)$ and $g\left(H \cap x_{\alpha}\right) \sim b$ are $\eta$-infinite, so $g\left(x_{\alpha}\right) \neq b$.

Assume $s \subset\{3,5\}$. Then $g(H) \subset b_{3} \cup b_{5}$. Consider all triples $\langle\delta, g(\delta), f g(\delta)\rangle$ where $\delta \in H$. Then by TUCA there is an $\eta$-infinite $H^{\prime} \subset H$ so that $\delta, g(\delta)$ and $f g(\delta)$ are all elements of $x_{\alpha}$ if $\delta \in H^{\prime}$. But then if $\delta \in H^{\prime}, g(\delta) \notin b_{3}$, since $b_{3} \subset \sim x_{\alpha} ;$ and $g(\delta) \notin b_{5}$, since $b_{5} \subset \sim f\left(x_{\alpha}\right)$ and $g(\delta)=f f g(\delta) \in f\left(x_{\alpha}\right)$. So $g\left(x_{\alpha}\right) \sim b$ is $\eta$-infinite.

Similarly, if we assume $s \subset\{2,4\}$ we conclude that $b \sim g\left(x_{\alpha}\right)$ is $\eta$-infinite, thus exhausting all the subsubcases of Subcase 1(a).

Subcase 1(b). $s \cap 6=\varnothing$. We do the subsubcase $s \subset\{6,7,9\}$; the others are handled similarly and are left to the reader.

Assume $s \subset\{6,7,9\}$, i.e., $g(H) \subset b_{6} \cup b_{7} \cup b_{9}$. Consider all triples $\langle\delta, g(\delta), f g(\delta)\rangle$ where $\delta \in H$. Then by TUCA there is an $\eta$-infinite $H^{\prime} \subset H$ so $\delta \in x_{\alpha}, g(\delta) \notin x_{\alpha}$, and $f g(\delta) \in x_{\alpha}$ for all $\delta \in H^{\prime}$. But then $g(\delta) \in f\left(x_{\alpha}\right) \sim x_{\alpha}$ if $\delta \in H^{\prime}$. By the $f-x$ canonical form, if its then $b_{i} \cap\left[f\left(x_{\alpha}\right)-x_{\alpha}\right]=\varnothing$, so if $\delta \in H^{\prime}$ then $g(\delta) \notin b$. Furthermore, $g\left(H^{\prime}\right) \subset$ $g\left(x_{\alpha}\right)$. So $g\left(x_{\alpha}\right) \sim b$ is infinite.

Case 2. $E \cap F$ is $\eta$-finite. Let $\overline{E \cap F}$ be the closure of $E \cap F$ under $g, g^{-1} . \overline{E \cap F}$ is an $\eta$-finite element of $M_{\alpha}$. Define $E^{*}=E \sim$ $\overline{E \cap F}, F^{*}=F \sim \overline{E \cap F}$. Note that $g$ is the identity on $E^{*}, g=f$ on $F^{*}$, and $E^{*} \cap F^{*}=\varnothing$.

Subclaim. Suppose $A=E^{*}$ off an $\eta$-finite set, or $A=F^{*}$ off an $\eta$-finite set. Then $A \notin B$.

Proof of subclaim. If $A \in B$, so is $\lambda-A$. Wlog assume $A=$ $F^{*}$ off an $\eta$-finite set. Then $g=f_{A}$ off an $\eta$-finite set, $f_{A} \in \mathscr{F}(\delta, B)$. This contradicts our hypothesis on $g$.

Returning to Case 2, if $\lambda-b^{*}$ is $\eta$-infinite then $g(x) \neq b$ by TUCA, so $\lambda-b^{*}$ is $\eta$-finite. Hence there is an $\eta$-finite $H \in M_{\alpha}$ and a finite $s \subset 10$ so that if $\delta \in H$ then $\psi(\delta)=x$, and both $H \cap E^{*}$, 
$H \cap F^{*}$ are $\eta$-infinite. (The second assertion follows from the subclaim-otherwise each Boolean combination of the $b_{i}^{*}$ 's would be contained $\bmod \eta$-finite in exactly one of $E^{*}$ or $F^{*}$. But then $E^{*} \bmod$ $\eta$-finite would be a finite union of sets in $B$, hence in $B$, which contradicts the claim.)

By TUCA, $s \neq\{1\}$. And by TUCA the following sets are $\eta$ in finite:

(i ) $\left\{g(\delta) \in H \cap E: \delta \in x_{\alpha}, g(\delta) \in x_{\alpha} \cap f\left(x_{\alpha}\right)\right\}$.

(ii) $\left\{g(\delta) \in H \cap E: \delta \in x_{\alpha}, g(\delta) \in x_{\alpha} \sim f\left(x_{\alpha}\right)\right\}$.

(iii) $\left\{g(\delta) \in H \cap E: g(\delta) \in x_{\alpha} \sim f\left(x_{\alpha}\right), \delta \in x_{\alpha}\right\}$.

(iv) $\left\{g(\delta) \in H \cap F: \delta \in x_{\alpha}, g(\delta) \in f\left(x_{\alpha}\right) \sim x_{\alpha}\right\}$.

Then by TUCA (i) shows that $g\left(x_{\alpha}\right) \sim b$ is $\eta$-infinite if $s \subset\{2,4,7,8,9\}$; (ii) shows that $g\left(x_{\alpha}\right) \sim b$ is infinite if $s \subset\{3,4,6,8,9\}$; (iii) shows that $b \sim g\left(x_{\alpha}\right)$ is $\eta$-infinite if $7 \in s$ or $s \subset\{3,5\}$; and (iv) shows that $g\left(x_{\alpha}\right) \sim b$ is $\eta$-infinite if $s \subset\{2,5,6,7,9\}$. By the $f-x$ canonical form this covers all possibilities, and Proposition 2.1 is proved.

The proof of Theorem 1 sketched in $\S 0$ is completed by noting that each $x_{\alpha} \sim f\left(x_{\alpha}\right)$ is an infinite element of $B$ which is moved by the automorphism $f$ onto a disjoint element.

5. Direct limits. To complete the proof of Theorems $3(\mathrm{~b})$ and 3 (c) we will combine the algebras constructed via the methods of 2 and 4 using a direct limit.

Definition 5.1. Let $S$ be a collection of disjoint subsets of $\lambda$ and for $A \in S$ let $B_{A}$ be an algebra on $A$. The direct limit $\sum_{A \in S} B_{A}$ is the algebra on $\lambda$ generated by $\left\{b \subset \lambda: b \in \bigcup_{A \in S} B_{A}\right\}$.

Note that $A \in S$ then $A \in \sum_{A \in S} B_{A}$.

Lemma 5.2. Let $B=\sum_{A \in S} B_{A}$ as in 5.1. Then if each $B_{A}$ has size $\kappa_{A}$ and exactly $\gamma_{A}$ automorphisms, $B$ has size $\sum_{A \in S} \kappa_{A}$ and at least $\Pi_{A \in S} \gamma_{A}$ automorphisms.

Proof. Size is clear. For the lower bound in automorphisms: suppose $g_{A}$ is an automorphism of $B_{A}$, for each $A \in S$. Then $\bigcap_{A \in S} g_{A}$ is an automorphism of $B$.

To make the lower bound on automorphisms an upper bound as well, we will take the direct limit of algebras constructed in parallel via the same Cohen objects. For orientation, we give an extremely rough description of how this is done.

Assume CA. Start out in the ground model with a collection of algebras $\left\{B_{A}: A \in S\right\}$ as in 5.1, and to each algebra associated a permutation $f_{A}$ of $A$. The sizes of the $A$ 's and $B_{A}$ 's may vary, as 
long as they are $\eta$-infinite, and as long as all of their elements are $\eta$-infinite. Then we take a collection of $\eta$-Cohen generic abjects, intersect them with the $A^{\prime}$ 's, and add these to our algebras, closing under the $f_{A}$ 's. Finally we take the direct limit. Imitation of previous arguments will show that the only automorphisms are those in the proof of 5.2, or variant moving a finite number of atoms.

We define the generic direct limit assumption.

GDL. Assume CA. Let $\eta \leqq \lambda<\rho$. Assume that the following hold in the ground model $M$ :

(1) $S$ is a partition of $\lambda$ into $\eta$-infinite sets.

(2) $\left\{f_{A}: A \in S\right\}$ is a family of functions, where each $f_{A} \in A$ ! and either $f_{A}$ is nice or it is the identity.

(3) $\{B(A): A \in S\}$ is a collection of algebras, each $B(A)$ is an algebra on $A, B(A)$ has size $>\lambda$.

(4) for each $A \in S, \kappa_{A}$ is the size of $B(A)$ and $\gamma_{A}$ is the number of automorphisms of $B(A)$.

(5) each infinite element of $B(A)$ is $\eta$-infinite.

Since by $1.2(\mathrm{v}), N$ is the model generated by $\left\{M_{\alpha}: \alpha<\lambda^{+}\right\}$where $x_{\alpha} \in M_{\alpha+1}$ is an $\eta$-Cohen subset of $\lambda$ over $M_{\alpha}$, in $N$ there is, for each $A \in S, B_{A}=$ the smallest $f_{A}$-closed algebra on $A$ containing $B(A) \cup$ $\left\{x_{\alpha} \cap A: \alpha<\lambda^{+}\right\}$. In $N$, let $B=\sum_{A \in S} B_{A}$.

That's the end of GDL. Note that every infinite element of $B$ is $\eta$-infinite.

Lenma 5.3. Assume GDL and suppose $g \in \lambda ! N$ moves an $\eta$ infinite set of atoms out of some $A \in S$. Then $g$ is not an automorphism of $B$.

Proof. For some $\alpha, g \in M_{\alpha}$ and $H \in M_{\alpha}$ where $H$ is an $\eta$-infinite subset of $A$ and $g(H) \cap A=\varnothing$. We claim that $g\left(x_{\alpha}\right) \notin B$.

For suppose $b \in B$. If $b$ is a subset of some finite union of elements of $S$, then the argument of 4.1 Case 1 applies directly. Otherwise, $b \supset A^{\prime}$ for some $A^{\prime} \in S$ and since, by TUCA, $g\left(x_{\alpha}\right)$ contains no $\eta$-infinite set in the ground model, we are done.

Lemma 5.4. Assume GDL, suppose $g$ does not fit the hypothesis of 5.3, and $g$ moves an infinite set of atoms out of some $A \in S$. Then $g$ is not an automorphism of $B$.

Proof. For some $\alpha, g \in M_{\alpha}$ and there is an infinite $\eta$-finite $H \subset A$, $H \in M$, with $g(H) \cap A=\varnothing$. By the argument of 2.1, if $g$ is an automorphism of $B$ then $g\left(x_{\beta} \cap A\right)=x_{\beta} \cap A$ for all $\beta \geqq \alpha$, but the density argument shows that for many $\beta \geqq \alpha g\left(x_{\beta} \cap A\right) \cap g(H) \neq \varnothing$, 
so $g\left(x_{\beta} \cap A\right) \sim\left(x_{\beta} \cap A\right) \neq \varnothing$ and we are done.

Putting together 5.2, 5.3, and 5.4 gives us

Corollary 5.5. Assume GDL. B has exactly $\prod_{A \in S} \gamma_{A}$ automorphisms.

Now we prove Theorem $3(b)$ and give a better version of $3(\mathrm{c})$.

Proof of 3(b). Assume CA and let $\lambda, \delta, \kappa$ be as in the hypothesis of $3(\mathrm{~b})$. Assume GDL where $S=\left\{A_{0}, A_{1}\right\},\left|A_{0}\right|=\left|A_{1}\right|=\lambda, B_{A_{0}}$ is constructed as in 4.1 with $\gamma_{A_{0}}=\kappa_{A_{0}}=\gamma$, and $B_{A_{1}}$ is constructed as in 2.1 to be almost rigid of size $\kappa$. Corollary 5.5 completes the proof.

Improved Theorem 3(c). Assume CA and let $\eta \leqq \lambda<\rho$. For each $\alpha<\lambda$ let $\kappa_{\alpha} \in\left[\lambda^{+}, 2^{\lambda}\right]$ and let $\gamma_{\alpha} \in\left[\lambda, \kappa_{\alpha}\right]$. Then where $\kappa=\sum_{\alpha<\lambda} \kappa_{\alpha}$ and $\gamma=\Pi_{\alpha<\lambda} \gamma_{\alpha},(\lambda, \gamma, \kappa)$ is realized.

Proof. Assume GDL where $S=\left\{A_{\alpha}: \alpha<\lambda\right\}$, and each $B_{A_{\alpha}}$ has size $\kappa_{\alpha}$ and exactly $\gamma_{\alpha}$ automorphisms. By 5.5 we're done.

Question. Recall the definition of a rich algebra in $\S 0$. Must the number of automorphisms of a rich algebra be the cardinality of an infinite product? In particular, is $\aleph_{\omega}$ possible?

\section{REFERENCES}

1. E. van Douwen, A consistent very small Boolean algebra with a countable automorphism group, (preprint).

2. E. van Douwen, J. D. Monk, and M. Rubin, Some questions about Boolean algebras, preprint.

3. J. de Groot and R. H. McDowell, Autohomeomorphism groups of zero-dimensional spaces, Compos. Math., 15 (1963), 203-209.

4. T. J. Jech, Set Theory, Academic Press, New York, 1978.

5. J. Loats, Hopfian Boolean algebras of power less than or equal to the continuum, Proc. Amer. Math. Soc., (to appear).

6. J. Loats and J. Roitman, Semi-rigid Hopfian and dual Hopfian atomic Boolean algebras, preprint.

7. R. McKenzie and J. D. Monk, On automorphism groups of Boolean algebra, Coll. Math. Soc. János Bolyai 10, Infinite and finite sets, Keszthely (Hungary), (1973), 951-988.

Received October 3, 1979 and in revised form June 26, 1980. This research was partially supported by NSF grants number MCS78-01851 and MCS-7702046 A01.

UNIVERSITY OF KANSAS

LAWRENCE, KS 66045 


\section{PACIFIC JOURNAL OF MATHEMATICS}

\section{EDITORS}

DONALD BABBITT (Managing Editor)

University of Galifornia

Los Angeles, California 90024

Hugo RossI

University of Utah

Salt Lake City, UT 84112

C. C. MOORE AND ANDREW OGG

University of California

Berkeley, CA 94720

\section{J. DugundjI}

Department of Mathematics University of Southern California

Los Angeles, California 90007

R. Finn AND J. Milgram

Stanford University

Stanford, California 94305

\section{ASSOCIATE EDITORS}

R. ARENS

E. F. BECKENBACH

B. H. NeUmanN

F. WOLF

K. YosHIDA

\section{SUPPORTING INSTITUTIONS}

UNIVERSITY OF ARIZONA

UNIVERSITY OF BRITISH COLUMBIA

CALIFORNIA INSTITUTE OF TECHNOLOGY

UNIVERSITY OF CALIFORNIA

MONTANA STATE UNIVERSITY

UNIVERSITY OF NEVADA, RENO

NEW MEXICO STATE UNIVERSITY

OREGON STATE UNIVERSITY
UNIVERSITY OF OREGON

UNIVERSITY OF SOUTHERN CALIFONIA

STANFORD UNIVERSITY

UNIVERSITY OF HAWAII

UNIVERSITY OF TOKYO

UNIVERSITY OF UTAH

WASHINGTON STATE UNIVERSITY

UNIVERSITY OF WASHINGTON 


\section{Pacific Journal of Mathematics}

\section{Vol. 94, No. 1 \\ May, 1981}

Willy Brandal, Conditions for being an FGC domain $\ldots \ldots \ldots \ldots \ldots \ldots \ldots$

Allan Calder and Frank Williams, Incompressibility of maps and the

homotopy invariance of Čech cohomology $\ldots \ldots \ldots \ldots \ldots \ldots \ldots \ldots \ldots$

Jacques Chaumat, Quelques propriétés du couple d'espaces vectoriels

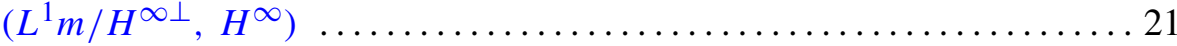

Manfred Droste and Rüdiger Göbel, Products of conjugate permutations . . 47

Jean Esterle, Rates of decrease of sequences of powers in commutative

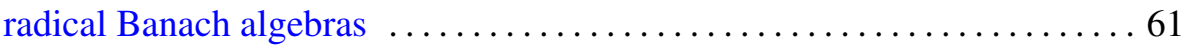

Allan Fryant, Ultraspherical expansions and pseudo analytic functions . . . 83

John Hannah, Homogenization of regular rings of bounded index. II . . . . . 107

Shigeru Haruki, On the theorem of S. Kakutani-M. Nagumo and J. L. Walsh

for the mean value property of harmonic and complex polynomials $\ldots .113$

Hugh M. Hilden, Representations of homology 3-spheres $\ldots \ldots \ldots \ldots \ldots 125$

Craig Huneke, A characterization of locally Macaulay completions . . . . . 131

Takesi Isiwata, Closed ultrafilters and realcompactness ................. 139

Joseph Weston Kitchen, Jr. and David A. Robbins, Tensor products of

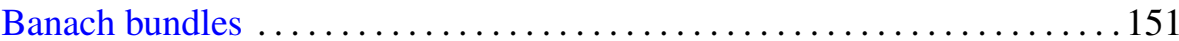

Allan J. Kroopnick, Note on bounded $L^{p}$-solutions of a generalized Liénard

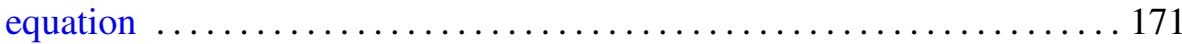

Ajay Kumar and Ajit Kaur Chilana, Spectral synthesis in products and quotients of hypergroups

Charles Livingston, Homology cobordisms of 3-manifolds, knot

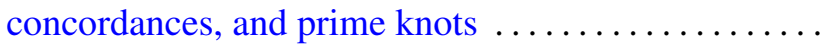

Hans Opolka, Projective representations of finite groups in cyclotomic fields

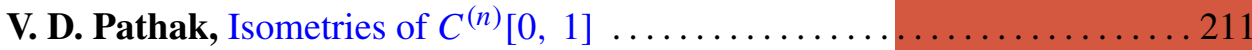

Mark Allan Pinsky, On the spectrum of Cartan-Hadamard manifolds . . . . . 2223

Judith Roitman, The number of automorphisms of an atomic Boolean

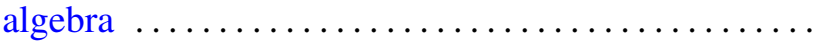

Kai Wang, Locally smooth torus group actions on integral cohomology complex projective spaces 